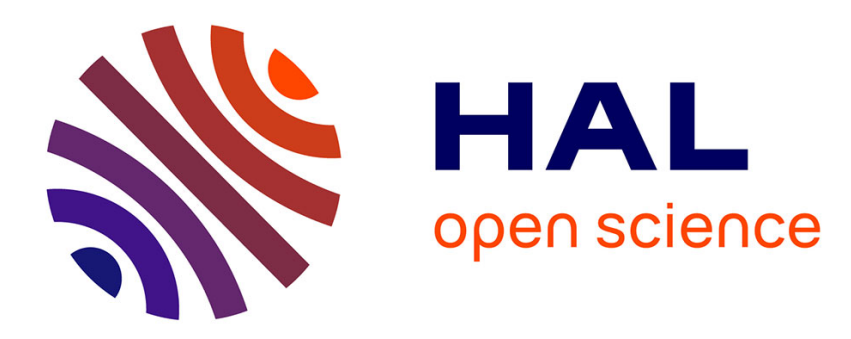

\title{
Les phénotypes des allergies respiratoires
}

Jocelyne Just

\section{To cite this version:}

Jocelyne Just. Les phénotypes des allergies respiratoires . Revue francaise d'allergologie, 2015, 55 (3), pp.108-109. 10.1016/j.reval.2014.12.003 . hal-01117095

\section{HAL Id: hal-01117095 \\ https://hal.sorbonne-universite.fr/hal-01117095}

Submitted on 16 Feb 2015

HAL is a multi-disciplinary open access archive for the deposit and dissemination of scientific research documents, whether they are published or not. The documents may come from teaching and research institutions in France or abroad, or from public or private research centers.
L'archive ouverte pluridisciplinaire HAL, est destinée au dépôt et à la diffusion de documents scientifiques de niveau recherche, publiés ou non, émanant des établissements d'enseignement et de recherche français ou étrangers, des laboratoires publics ou privés. 


\section{Les phénotypes des allergies respiratoires}

\section{Allergic phenotypes in children}

J Just

${ }^{1}$ Service D'Allergologie pédiatrique, Centre de 1'Asthme et des Allergies. Hôpital d'Enfants Armand-Trousseau (APHP) -

${ }^{2}$ Sorbonne Universités, UPMC Univ Paris 06, UMR_S 1136, Institut Pierre Louis d'Epidémiologie et de Santé Publique, Equipe EPAR, F-75013, PARIS 75571 Cedex

Adresse pour correspondance

\section{Pr. Jocelyne JUST}

Centre de 1'Asthme et des Allergies. Hôpital d'Enfants Armand-Trousseau (APHP) - 26, Avenue du Dr. Arnold Netter, 75571 PARIS Cedex 12. France.

Tel. +33144736317

Fax: +33144736635

E-mail: jocelyne.just@ trs.aphp.fr

Mots clés : Phénotype d'asthme, allergie, eczéma, sensibilisation allergénique

Key words: asthma phenotype, allergy, eczema, allergic sensitization

L'asthme est classiquement défini comme une pathologie chronique inflammatoire des voies aériennes. Cette maladie est caractérisée par une hyperréactivité des voies aériennes et une obstruction bronchique réversible. Elle affecte 2-300 000000 personnes dans le monde et sa prévalence a augmenté au cours des dernières décennies. Typiquement, l'inflammation dans l'asthme est décrite comme étant allergique, éosinophilique, dépendante des IgE, et Th2 médié. Cependant plus de 50\% de patients asthmatiques n'ont pas d'inflammation éosinophilique ${ }^{1}$. Aussi, la notion d'une physiopathologie uniciste classique a été remise en question et l'hypothèse de voies physiopathologiques différentes responsables de différents phénotypes de l'asthme s'est développée.

La stabilité des phénotypes de l'asthme chez les très jeunes enfants dépend de l'expression de l'allergie. Plusieurs scores ont été développés pour prédire la persistance de l'asthme durant l'enfance comme le Predictive Index asthma (API) dans la cohorte Tucson ou encore un score provenant de la cohorte PIAMA. Toutefois, ces règles de prédiction sont difficiles à appliquer dans la pratique clinique en raison d'une faible valeur prédictive positive. 
Une autre approche de ce problème est d'étudier si les phénotypes d'asthme définis prospectivement pourraient avoir une trajectoire différente pendant l'enfance et donc améliorer la prévision de l'évolution de l'asthme. De cette manière, la cohorte de TAP a révélé un bon pronostic pour les enfants appartenant au phénotype asthme viro-induit léger: à 5 ans, $69 \%$ des enfants appartenant à ce phénotype présentaient un asthme léger ou étaient en rémission ${ }^{2}$. Dans cette même cohorte, aucun des enfants du phénotype "atopique à facteurs déclenchant multiples" n'était asymptomatique à l'âge de 5 ans. Spycher et al. ${ }^{3}$ ont trouvé un phénotype intermédiaire entre l'asthme allergique persistant et le viro-induit léger en terme de pronostique à l'adolescence. Ce phénotype pourrait être associé à un asthme persistant dans l'enfance en relation avec l'apparition d'une allergie tardive.

Plus globalement, la leçon à retenir sur le pronostic de l'asthme dans l'enfance est l'évolution des phénotypes ne dépend pas seulement de l'expression de l'allergie, mais aussi du moment de l'apparition de l'allergie (précoce ou tardive).

\section{Chez l'enfant, l'expression de l'allergie définit le pronostic de sévérité de l'asthme au} cours de l'enfance et probablement durant toute la vie, mais le problème est de savoir comment l'allergie devrait-elle être définie?

L'allergie peut se définir (1) comme une sensibilisation allergique (simple ou multiple) où il faudrait prendre en compte le type d'allergène en cause et la date d'apparition de la sensibilisation allergènique (2) et/ou encore par l'association des comorbidités allergiques (comme la rhinite allergique, l'eczéma ou les allergies alimentaires).

L'apparition d'une sensibilisation allergique tôt dans la vie est un facteur de risque important pour le développement de l'asthme. Plus particulièrement, des sensibilisations aux aéroallergènes multiples et précoces sont à risque accru de développement d'asthme infantile ${ }^{4}$. Les cohortes néonatales $\mathrm{CAPS}^{5}$ et $\mathrm{MAAS}^{6}$ définissent une population d'enfants monosensibilisés aux acariens qui confère un meilleur

pronostic de l'asthme par rapport aux enfants polysensibilisés. Une étude provenant des cohortes $\mathrm{TAP}^{7}$, réalisée dans 125 enfants d'âge scolaire atteints d'asthme allergique, décrit quatre phénotypes d'asthme allergique et spécialement un phénotype d'asthme léger avec une monosensibilisation aux acariens dans $98 \%$ des cas. Dans une étude précédente, une autre cohorte TAP décrit un phénotype similaire d'asthme léger ${ }^{8}$.

A l'inverse dans la cohorte MAAS $^{9}$, les enfants (surtout les garçons) présentant une multisensibilisation allergénique sont plus à risque d'exacerbations d'asthme fréquentes et multiples et également de perte progressive de la fonction pulmonaire entre l'âge de 3 ans à 
11 ans. De la même façon Garden et al. ${ }^{10}$ ont montré que le risque d'asthme était lié au type de la sensibilisation allergénique, avec une forte association entre les sensibilisation alimentaire mixte (pneumallergènes et trophallergènes) et le mauvais contrôle de l'asthme à l'âge de 8 ans.

L'étude multicentrique allergie (MAS) représente une cohorte allemande qui se compose de 1314 enfants suivis tous les ans jusqu'à l'âge de 13 ans. Cette cohorte a constaté que l'eczéma associée à des mutations d'une protéine exprimée dans la peau (la filaggrine) était plus à risque d'asthme sévère associé à une diminution significative de la fonction pulmonaire à la puberté. De même, dans une étude portant sur une des cohortes TAP, un phénotype d'asthme sévère (associé à une diminution significative du DEM25-75) présente des manifestations allergiques multiples (100\% des enfants souffrent d'eczéma et sensibilisations allergéniques multiples).

Un autre phénotype de l'asthme allergique sévère à l'âge scolaire a été décrit dans une des cohortes TAP $^{9}$. Ce phénotype était représenté dans par des enfants qui avaient dans $92 \%$ des cas des antécédents d'exacerbations graves associés à une sensibilisation aux pollens. Erbas et $\mathrm{al}^{11}$ ont montré une augmentation linéaire de venues aux urgences pour des exacerbations aigues d'asthme en corrélation avec une concentration accrue dans l'air de pollens de graminées $(\mathrm{p}<0,001)$.

Pour résumer, l'asthme allergique sévère chez les enfants pourrait se composer de deux phénotypes allergiques en fonction du type de la sensibilisation et de l'association avec d'autres comorbidités allergiques: un phénotype sévère constamment associé à un eczéma avec des allergies multiples et un autre phénotype sévère présentant des exacerbations aiguës liées à l'exposition aux pollens. 
${ }^{1}$ McGrath KW, Icitovic N, Boushey HA, et al. Asthma Clinical Research Network of the National Heart, Lung, and Blood Institute. A large subgroup of mild-to-moderate asthma is persistently noneosinophilic. Am J Respir Crit Care Med 2012;185:612-9.

${ }^{2}$ Just J, Saint-Pierre P, Gouvis-Echraghi R, et al.. Wheeze phenotypes in young children have different courses during the preschool period. Ann Allergy Asthma Immunol. 2013; 111: 256261.

${ }^{3}$ Spycher BD, Silverman M, Brooke AM, et al. Distinguishing phenotypes of childhood wheeze and cough using latent class analysis. Eur Respir J. 2008; 31: 974-81.

${ }^{4}$ Simpson A, Tan VY, Winn J, et al. Beyond atopy: multiple patterns of sensitization in relation to asthma in a birth cohort study. Am J Respir Crit Care Med 2010;181:1200-6.

5 Mihrshahi S, Peat J, Webb K, et al. The Childhood Asthma Prevention Study (CAPS): design and research protocol of a large randomised trial of the primary prevention of asthma. Control Clin Trials 2001; 22:333-54.

${ }^{6}$ Belgrave DC, Buchan I, Bishop C, et al. Trajectories of lung function during childhood. Am J Respir Crit Care Med. 2014; 189: 1101-1109.

${ }^{7}$ Just J, Saint-Pierre P, Gouvis-Echraghi R, et al. Childhood allergic asthma is not a single phenotype. J Pediatr. 2014; 164: 815-820.

${ }^{8}$ Just J, Gouvis-Echraghi R, Rouve S, et al. Two novel, severe asthma phenotypes identified during childhood using a clustering approach. Eur Respir J 2012; 40: 55-60.

${ }^{9}$ Belgrave DC, Buchan I, Bishop C, et al.. Trajectories of lung function during childhood. Am J Respir Crit Care Med. 2014; 189: 1101-1109.

${ }^{10}$ Garden FL, Simpson JM, Marks GB; CAPS Investigators. Atopy phenotypes in the Childhood Asthma Prevention Study (CAPS) cohort and the relationship with allergic disease: clinical mechanisms in allergic disease. Clin Exp Allergy. 2013; 43: 633-641.

${ }^{11}$ Erbas B, Akram M, Dharmage SC, et al. The role of seasonal grass pollen on childhood asthma emergency department presentations. Clin Exp Allergy 2012; 42: 799-805. 